\title{
Editorial
}

\section{Fangjiomics: revealing adaptive omics pharmacological mechanisms of the myriad combination therapies to achieve personalized medicine}

\author{
Dayue Darrel DUAN ${ }^{1, *}$, Zhong WANG ${ }^{2, *}$, Bo-li ZHANG ${ }^{3, *}$, Yong-yan WANG ${ }^{2, *}$ \\ ${ }^{1}$ Laboratory of Cardiovascular Phenomics, Department of Pharmacology, Center for Molecular Medicine, University of Nevada School of \\ Medicine, Reno, Nevada 89557, USA; ${ }^{2}$ Institute of Basic Research in Clinical Medicine, China Academy of Chinese Medical Sciences, \\ Beijing 100700, China; ${ }^{3}$ Tianjin University of Traditional Chinese Medicine, Tianjin 300193, China
}

Acta Pharmacologica Sinica (2015) 36: 651-653; doi: 10.1038/aps.2015.33

In Western medicine, therapeutic drugs for the treatment of diseases are required to have a well-defined pure molecular target (one protein or one gene) because the understanding of molecular mechanisms for diseases are largely based on the principle of phenotype-genotype relationship, ie, "one gene encoding one protein and responsible for one phenotype" ${ }^{\text {[1] }}$. In clinical practice, however, treatment of many diseases often requires a multi-drug regimen that combines several monotherapies targeting on different molecules to optimize pharmacodynamics and/or pharmacokinetics for the purpose of improving therapeutic efficacy and/or reducing toxicity and adverse reactions ${ }^{[2]}$. Recent findings from studies of pharmacogenomics and the newly-developed pharmacoproteomics ${ }^{[3]}$ and pharmacometabolomics ${ }^{[4]}$ have revealed pervasive effects of genetic polymorphisms on drug efficacy or toxicity, not only urging for personalized drug treatments according to individual patient's genetic characteristics but also adding more layers of complexity to the combination of multi-drug regimen ${ }^{[4]}$. Furthermore, the current definition of clinical phenotypes of disease ( $e g$, hypertension, heart failure, type-2 diabetes, etc) and related drug therapy has shown serious limitations that conceal the ever-increasing details of genomic variants discovered by the genome-wide association studies $(G W A s)^{[5]}$. Clearly, newer approaches are needed for Western medicine to refine the symptoms- or evidence-based definition

\footnotetext{
* To whom correspondence should be addressed.

E-mail dduan@medicine.nevada.edu (Dayue DUAN); zhonw@sina.com (Zhong WANG); zhangbolipr@163.com (Bo-li ZHANG); wangyongyan2010@sina.cn (Yong-yan WANG)

Received 2015-02-12 Accepted 2015-03-17
}

of disease and drug responses so that the intrinsic complexity of the disease, the outcomes of drug treatment, and the impact of multiple environmental factors can be unequivocally characterized and classified. Obviously, this new approach for the redefinitions of disease and drug response (efficacy and toxicity) has to be at the "ome"-level to encompass a big range of symptomatic, morphological, biological, pathophysiological, behavioral, genomic, proteomic, and metabolomic features.

In the traditional Chinese medicine (TCM), fundamentally different from the Western medicine, diseased phenotypes are clustered as Zheng-hou and treated with a combination of several herbs called Fangji. While the empiric based Fangji therapy of Zheng-hou has been proven clinically effective, the underlying mechanisms for the combined targets remain mysteries.

Fangjiomics is recently emerged as a new discipline to systematically study myriad compatible combinations that may act through multiple targets and modes of actions balancing on-targets with off-targets ${ }^{[6]}$. Different mechanisms and dynamic characteristics of combination therapies are delineated through collecting diverse evidences and analyzing the designs of optimal patterns of Fangji therapy from the long history of clinical practice and literature. Fangjiome is composed of thousands of Fangjis. A variety of omics technologies and related analytical tools are combined to reveal the complex relationships in the Fanjiome at different omics levels. By prioritizing targets, pathways and ingredient spectra, Fangiiomics may lead to the discovery of controllable array-designed therapies to combine less potent elements with more on-target effects and fewer off-target effects than monotherapy. 
Based on archives of a Fang-Zheng Forum held in Beijing, China on November 24-25, 2013, we have assembled in this Special Issue a series of original research and review articles of Fangjiomic studies on several popular Fangjis in different sizes with diverse compositions of numbers of herbs or compounds. These papers represent some of the significant progress in Fangjimoics in recent years and its impact on not only TCM but also the application of systems biology, network pharmacology, and bioinformatics to the current translational medicine.

To rationally translate the studies of Fangjiomics into clinical practice, we should know clearly the relationship of multiple compounds and related effects. So a key concept of Fangjiomics is to highlight the effect-based compatibility principle of Chinese medicinal prescription $(\text { Fangji })^{[7]}$. In this regard, Li et al reviewed Fangjis of promoting blood circulation for removing blood stasis (PBCRBS) therapy for acute intracerebral hemorrhage patients. PBCRBS could ameliorate neurological function deficits, reduce volume of hematoma and volume of perihematomal edema, and lower mortality rate and dependency in comparison with Western conventional medication controls or placebo based on a Meta-analysis ${ }^{[8]}$. Wang et al summarized the recent investigations on the anticancer activities and possible molecular targets of the Compound Kusen Injection, and then hypothesized that altering gene regulation in several pathways including Wnt signaling pathway might be involved in the underlying mechanisms $\mathrm{s}^{[9]}$. To summarize the targets distribution of Fangjis, Liu and Wang defined multiple modes of array-designed combination therapies of Fangjiomics, such as "magic shotguns", vertical, horizontal, focusing, siege and dynamic arrays according to hits on targets, pathways or networks ${ }^{[10]}$.

Also included in this Special Issue are six original research articles that delineate the systematic and network approaches of Fangjiomics on several popular Zheng-hous or Fangjis, which are commonly used in TCM practice. A proteomic and metabolomic profiling of Kidney-Yin Deficiency Syndrome (KYDS) (Zheng-hou) patients with diabetes mellitus not only detected for the first time the potential biomarkers associated with KYDS, but also provided compelling evidence for the existence of a molecular basis in KYDS syndrome differentiation $^{[11]}$. The network-based approach illustrated the complex therapeutic mechanisms of Xiao-Ke-An for type 2 diabetes through improving the carbohydrate and lipid metabolisms, ameliorating insulin resistance, attenuating diabetic vascular complications, and anti-inflammation ${ }^{[12]}$. Wang et al demonstrated that Chang'an II protected the intestinal mucosa against post-inflammation irritable bowel syndrome (PI-IBS) through integrated effects of immunomodulation, anti-inflammation, and anti-anxiety ${ }^{[13]}$.

In two articles by Zhao et al ${ }^{[14]}$ and Zhang et al ${ }^{[15]}$, the contributions of different parties in Fangjis to the total action of Fangjis were demonstrated. Though each single herb (Ramulus Cinnamomi, Guizhi; Paeonia lactiflora, Shaoyao; Rhizoma Anemarrhenae, Zhimu) could affect some diabetic peripheral neuropathy (DPN)-related functions and pathways, Guizhi-
Shaoyao-Zhimu decoction showed more effects on those DPNrelated functions and pathways, such as aryl hydrocarbon receptor signaling and apoptosis signaling ${ }^{[14]}$. A network analysis of the ingredients in Huanglian-Jie-du Decoction revealed their putative targets in ischemic stroke-related pathway systems ${ }^{[15]}$. With a rational combination of compounds diverse functions with different combined modes may emerge for the therapeutic purpose. For example, ursodeoxycholic acid and jasminoidin in the Qingkailing injection yielded ten new core pathways involving immune responses, apoptosis, and nervous system, etc. for the treatment of stroke and brain ischemia/reperfusion. This study provided new insight into the combination therapy of pure synergism ${ }^{[16]}$.

Dose alterations result in variations of Fangji's action. Guo et al demonstrated that overlapping and non-overlapping metabolites and metabolic pathways provided a dose-dependent metabolic mechanism for the neuroprotective effects of DanHong injection in the treatment of cerebral ischemia ${ }^{[17]}$.

The composition of herbs Fangjis is different in size. The simplest Fangji has only a single herb or compound and is in the category of Dan-fang. Liu et al studied the effect of ginsenoside Rd on neurogenesis after focal cerebral ischemia/ reperfusion injury (IRI) in rats and its underlying mechanisms. Even a single compound, Rd promoted neurogenesis after IRI by upregulating VEGF and BDNF expression through PI3K/ Akt and ERK1/2 pathways ${ }^{[18]}$. Similarly, tannins fraction (PTFs) could induce apoptosis and inhibit the migration and invasion of NCI-H1703 cells via regulating the MMPs expression through MAPK/MMPs pathways in human lung squamous carcinoma cells ${ }^{[19]}$.

These significant advances in application of the concepts and research approaches of Fangjimocs in the study of the adaptive regulation and hierarchical architectural theory of Fangji's integrated and balanced paradigm of multiple targets, such as Jun-Chen-Zuo-shi, provide preliminary promise for Fangjiomics as a novel and effective approaches to the development of combination therapy in different diseases. In order to reveal the interconnections of multiple compounds, complex pathways and fluctuant networks of diseases and drug responses, we need to 1) understand how any alteration of Fangjis results in variations of Zheng-hou and drug response and treatment outcomes; and 2) reveal more and more pharmacological mechanisms of different Fangjis in the treatment of corresponding Zheng-hou.

In many aspects, Fangjiomics is in common with pharmacophenomics ${ }^{[20]}$ in Western medicine. If we would define Zhenghou as the disease phenome, Fangjiomics would provide clues and ideas for pharmacophenomics in identification of the corresponding therapeutic targets and drug responses. The translation of the knowledge of pharmacogenomics and pharmacophenomics etc. into clinical application of Fangjiomics would pave a new path to deconstructing the target networks and disease pathological pathways and help to understand the crosstalk of these pathways or sub-networks and to design optimal and perfect combination of multiple herbs or compounds that is effective to reverse those related pathways or 
networks ${ }^{[21]}$. Therefore, we expect that the continuous application of Fangjiomics in the evaluation of more effective Fangjis on Zheng-hou may provide more solid basis for personalized medicine ${ }^{[22]}$ in near future.

\section{References}

1 Schork NJ. Genetics of complex disease: approaches, problems, and solutions. Am J Respir Crit Care Med 1997; 156: S103-9.

2 Nebert DW, Zhang G, Vesell ES. From human genetics and genomics to pharmacogenetics and pharmacogenomics: past lessons, future directions. Drug Metab Rev 2008; 40: 187-224.

3 Wang Z, Duan DD, Wang YY. Combination therapy of vascular diseases and Fangjiomics: when West meets East in the era of phenomics. Curr Vasc Pharmacol 2014. Doi: 10.2174/15701611126 66141013114052.

4 Kaddurah-Daouk R, Weinshilboum RM. Pharmacometabolomics: implications for clinical pharmacology and systems pharmacology. Clin Pharmacol Ther 2014; 95: 154-67.

5 Ye Z, Mayer J, Ivacic L, Zhou Z, He M, Schrodi SJ, et al. Phenome-wide association studies (PheWASs) for functional variants. Eur J Hum Genet 2015; 23: 523-9.

6 Wang Z, Liu J, Cheng YY, Wang YY. Fangjiomics: in search of effective and safe combination therapies. J Clin Pharmacol 2011; 51: 113251.

7 Zhang JH, Zhu Y, Fan XH, Zhang BL. Efficacy-oriented compatibility for component-based Chinese medicine. Acta Pharmacol Sin 2015; 36 : 654-8.

$8 \mathrm{Li} \mathrm{HQ}$, Wei JJ, Xia W, Li JH, Liu AJ, Yin SB, et al. Promoting blood circulation for removing blood stasis therapy for acute intracerebral hemorrhage: a systematic review and meta-analysis. Acta Pharmacol Sin 2015; 36: 659-75.

9 Wang W, You RL, Qin WJ, Hai LN, Fang MJ, Huang GH, et al. Antitumor activities of active ingredients in Compound Kushen Injection. Acta Pharmacol Sin 2015; 36: 676-9.

10 Liu J, Wang Z. Diverse array-designed modes of combination therapies in Fangjiomics. Acta Pharmacol Sin 2015; 36: 680-8.

11 Jiang N, Liu HF, Li SD, Zhou WX, Zhang YX, Zhang Q, et al. An integrated metabonomic and proteomic study on Kidney-Yin Deficiency Syndrome patients with diabetes mellitus in China. Acta Pharmacol
Sin 2015; 36: 689-98.

12 Yang ZZ, Liu W, Zhang F, Li Z, Cheng YY. Deciphering the therapeutic mechanisms of Xiao-Ke-An in treatment of type 2 diabetes in mice by a Fangjiomics approach. Acta Pharmacol Sin 2015; 36: 699-707.

13 Wang FY, Su M, Zheng YQ, Wang XG, Kang N, Chen T, et al. Herbal prescription Chang'an II repairs intestinal mucosal barrier in rats with post-inflammation irritable bowel syndrome. Acta Pharmacol Sin 2015; 36: 708-15.

14 Zhao N, Li J, Li L, Niu XY, Jiang M, He XJ, et al. Molecular networkbased analysis of Guizhi-Shaoyao-Zhimu decoction, a TCM herbal formula, for treatment of diabetic peripheral neuropathy. Acta Pharmacol Sin 2015; 36: 716-23.

15 Zhang YQ, Wang SS, Zhu WL, Ma Y, Zhang FB, Liang RX, et al. Deciphering the pharmacological mechanism of the Chinese formula Huanglian-Jie-Du decoction in the treatment of ischemic stroke using a systems biology-based strategy. Acta Pharmacol Sin 2015; 36: 724-33.

16 Wang PQ, Li B, Liu J, Zhang YY, Yu YN, Zhang XX, et al. Phenotypedependent alteration of pathways and networks reveals a pure synergistic mechanism for compounds treating mouse cerebral ischemia. Acta Pharmacol Sin 2015; 36: 734-47.

17 Guo ZL, Zhu Y, Su XT, Liu J, Yang QX, Nan JY, et al. DanHong injection dose-dependently varies amino acid metabolites and metabolic pathways in the treatment of rats with cerebral ischemia. Acta Pharmacol Sin 2015; 36: 748-57.

18 Liu XY, Zhou XY, Hou JC, Zhu H, Wang Z, Liu JX, et al. Ginsenoside $\mathrm{Rd}$ promotes neurogenesis in rat brain after transient focal cerebral ischemia via activation of PI3K/Akt pathway. Acta Pharmacol Sin 2015; 36: 421-8.

19 Zhao HJ, Liu T, Mao X, Han SX, Liang RX, Hui LQ, et al. Fructus phyllanthi tannin fraction induces apoptosis and inhibits migration and invasion of human lung squamous carcinoma cells in vitro via MAPK/MMP pathways. Acta Pharmacol Sin 2015; 36: 758-68.

20 Duan DD, Han YS, Li L, Zhao JZ, Wang Z. Pharmacophenomics: a new paradigm for pharmacology, toxicology, and personalized medicine. Chin J Pharmacol Toxicol 2014; 28: 1-9.

21 Flemming A. Finding the perfect combination. Nat Rev Drug Discov 2015; $14: 13$.

22 Wang Z, Wang YY. Navigating personalized medicine dependent on modular flexibility. Trends Mol Med 2013; 19: 393-5. 